\title{
TREE DROUGHT STRESS DETECTION BASED ON 3D MODELLING
}

\author{
Y. Xia, J. Tian, P. d'Angelo, P. Reinartz \\ German Aerospace Center (DLR), Remote Sensing Technology Institute, 82234 Wessling, Germany \\ - (yuanxin.xia, jiaojiao.tian, pablo.angelo, peter.reinartz)@dlr.de
}

\section{ICWG II/III: Pattern Analysis in Remote Sensing}

KEY WORDS: Tree, 3D Modelling, Semi-Global Matching, Convolutional Neural Networks, Drought Detection, Deformation

\begin{abstract}
:
Precise and detailed reconstruction of 3D plant models is an important goal in computer vision. Based on these models, important parameters can be extracted, which would be very useful for monitoring the tree health situation. This paper has firstly constructed the 3D plant model based on MC-CNN using close-range photogrammetric imagery, and then applied a leaf index based segmentation to highlight the leaves region. In the end, the 3D model of each leaf can be represented and some geometric parameters of the leaf are designed and analyzed to predict the drought status. The experiments on real close-range stereo imagery justified the performance of the proposed approach to differentiate drought and healthy leaves.
\end{abstract}

\section{INTRODUCTION}

Global warming increases the possibility of trees suffering from drought stress. The early detection of drought stress symptoms provides opportunities to take action and enhance the surviving ability of trees at a reversible stage. Such, the forest management can be better organized which is significant in numerous fields such as environment, politics, economics, climate and ecology (Strigul, 2012). Remote sensing is a concept to remotely measure the materials on the Earth surface by means of different imaging techniques (Hong et al., 2018), which makes it essentially appropriate to observe the forest and provide time series datasets for constant monitoring.

Recently, space- and air-borne remote sensing measurements have been widely used for forest management to estimate the biomass, monitor the living condition, measure the forest canopy cover, derive detailed digital surface models, etc. (Ahmed et al., 2014) (Freeman et al., 2016) (Wu et al., 2016) (Tian et al., 2017). Among different measuring targets, drought stress is an important factor which affects the functioning and recovery of the forest ecosystem ( $\mathrm{Xu}$ et al., 2018).

However with only these large scale properties, it is still not straightforward to perceive the drought stress, which inspires the need of constructing 3D models of single trees using close-range photogrammetric stereo imagery with high resolution. Based on precisely constructed 3D models, single tree growth patterns can be followed from intuitive geometric characteristics. Especially when considering the filigree leaves, the shape, size, color are all able to provide important information to determine the drought stress of the tree, so that the whole ecosystem can be better understood (Levin, 1999) (Gatziolis et al., 2015). However, until now most of the $3 \mathrm{D}$ tree modelling researches have been conducted based on LiDAR datasets, to estimate the plant scaling parameters (Lau Sarmiento et al., 2015), observe competition on tree growth (Metz et al., 2013), simulate radiative transfer models (Calders et al., 2018), etc.

$3 \mathrm{D}$ reconstruction based on stereo imagery is an important and interesting topic in computer vision. Along with the improved camera systems and computation techniques, reconstructing $3 \mathrm{D}$ models of small objects with limited images is becoming possible. However, the $3 \mathrm{D}$ reconstruction of plants, as well as segmentation of single leaves are still among the most challenging tasks in this filed.

Focusing on dense matching based 3D reconstruction, Semi-Global Matching (SGM) (Hirschmueller, 2008) is one of the most robust approaches in accuracy and efficiency. Recently, the convolutional neural networks $(\mathrm{CNN})$ has been introduced for the matching cost computation (Zbontar , LeCun, 2016), which performed well in plant reconstruction (Xia et al., 2019). In this paper, we have extracted single leaves from images which will be further integrated with the 3D plant model to distinguish leaves under drought stress.

\section{METHODOLOGY}

\subsection{MC-CNN based 3D Reconstruction}

CNN is essentially a feed-forward artificial neural network constructed by a sequence of layers with learnable weights and biases (LeCun et al., 1998). Unlike the hand-crafted feature extraction (Hong et al., 2015), CNN has been proven to be effective in automatically extracting discriminative features for a wide range of vision tasks, such as classification (Krizhevsky et al., 2012) (Hang et al., 2019), recognition (Bhandare et al., 2016), detection (Wu et al., 2018), etc. Zbontar and LeCun (2016) proposed to compute Matching Cost based on CNN (MC-CNN) for dense matching. They trained a siamese network on pairs of image patches with the true disparity known, to learn a similarity score (Bromley et al., 1993) (Zbontar, LeCun, 2016). Two architectures are designed, of which one concentrates on the efficiency by using a fixed similarity measure, named as fast architecture. The other network, accurate architecture, is a modified implementation of the first. It learns the similarity during training. Afterwards, SGM is exploited to aggregate the cost for disparity estimation. The proposed algorithm has outperformed 
many traditional stereo matching methods on KITTI (Geiger et al., 2013) (Menze , Geiger, 2015) and Middlebury (Scharstein , Szeliski, 2002) (Scharstein, Szeliski, 2003) (Scharstein , Pal, 2007) (Hirschmueller , Scharstein, 2009) (Scharstein et al., 2014) datasets.

The accurate architecture is adopted in this research to meet the high-quality demand of the tree leaves $3 \mathrm{D}$ reconstruction. The structure of the network is shown in Figure 1. However, there is no proper stereo matching dataset for plant reconstruction to train MC-CNN. Therefore, we follow our previous work and fine-tune a pre-trained network on Middlebury based on a self-training scheme (Xia et al., 2019). Starting from the standard weights of the pre-trained network, a disparity map is generated, from which most matching outliers are filtered according to a left-right consistency check. Regarding left and right image as the master epipolar frame, respectively, the pixels with the estimated disparity differing by less than one pixel are kept to fine-tune MC-CNN. Thus, the network is adaptively adjusted and capable of achieving higher performance for matching cost computation and further disparity prediction.

\subsection{Leaves-Covered Region Extraction}

In order to remove the influences from background, the Visible Atmospheric Resistant Index (VARI) which was developed at the university of Nebraska is used here to highlight the leaves region from RGB images (Kaufman, 1989) (Gitelson et al., 2002).

$$
V A R I=\frac{R_{\text {Green }}-R_{\text {Red }}}{R_{\text {Green }}+R_{\text {Red }}-R_{\text {Blue }}}
$$

The regions with VARI between 0.2 to 1 are kept to extract the leaves. Afterwards, we have removed the thin objects and small holes, and then dilated the large objects based on morphological features for better boundary recovery. Hence single-leaf segments are highlighted for further geometric analysis.

\subsection{Drought Stress Detection}

Based on our current experience, leaves with water shortage would be inclined to bend inwards to decrease the water-lose speed. Under this hypothesis, the drought status can be determined by computing the relative orientation of each sub-area of the leaf with respect to a reference plane, e.g. a fitting plane of the leaf's curved surface, as shown in Figure 2. A larger difference between the orientation indicates a higher possibility of the leaf to suffer from drought stress. In other words, the included angle between the normal vector of each sub-area and the normal vector of the reference plane can be calculated to study the curvature of the leaf, as well as the drought status.

\section{EXPERIMENT}

\subsection{Data}

Two digital high-resolution handheld cameras (SONY ILCE-5100) with $19 \mathrm{~mm}$ lens are mounted on a crane system in nadir-view for stereo measurement. The exposure time is set as 1/60 seconds and an ISO speed rating of 125 is used. When the system is lifted above the trees, a stereo image pair of the tree crown is obtained. The acquired images are
4000 and 6000 pixels respectively in height and width. The baseline of the stereo pair has a length of approximately 0.25 meters and the distance of the cameras from the tree crown is set as approximately 3 meters. In order to test the feasibility of the method described in this paper, a stereo image pair above a beech tree subject to slightly artificial drought stress is collected. Details about the images and the camera setting are recorded in Table 1.

\begin{tabular}{|l|c|}
\hline Camera model & SONY ILCE-5100 \\
\hline Height & 4000 pixels \\
Width & 6000 pixels \\
Exposure time & $1 / 60 \mathrm{sec}$ \\
ISO speed rating & 125 \\
Focal length & $19 \mathrm{~mm}$ \\
Baseline length & $\approx 0.25 \mathrm{~m}$ \\
Object distance & $\approx 3 \mathrm{~m}$ \\
\hline
\end{tabular}

Table 1. Details for acquiring the stereo image pair

\subsection{Dense Matching Results}

Before dense matching, MicMac (Rosu et al., 2015) is exploited for camera calibration, relative orientation and image rectification. Afterwards, SGM based on self-trained MC-CNN is implemented to generate a disparity map. The master epipolar image and the generated disparity map are shown in Figure 3. The color bar below is in units of pixels. From the color blue to red, the object distance is decreasing.

\subsection{Evaluation}

Based on the generated disparity map, the drought stress status of single leaves can be evaluated. Firstly, the leaves-covered region is extracted to remove the influence of the background, e.g. the branches, according to the calculated VARI. The extracted leaves area is shown in Figure 4.

Under the hypothesis that curled leaves are more possible to suffer from drought stress than planar ones, leaves with relatively curled surface are manually selected and compared with more planar leaves regarded as healthy. This procedure aims at testing the feasibility of the proposed processing scheme for differentiating curled and planar leaves. The leaf selection is supposed to be performed based on automatic segmentation algorithm. However for our dataset, the self-similarity and dense distribution of leaves highly increases the difficulty level to acquire a satisfying segmentation result. Besides, the training data generation is time-consuming for deep learning based approaches. Therefore, up to now, manual intervention is adopted.

The point cloud of each selected leaf is generated according to the calculated disparity which is then used for a Delaunay triangulation (Delaunay, 1934). Afterwards, the normal vectors of all tiny triangles are calculated and averaged to approximate the normal vector of the reference plane as mentioned in Section 2.3. Thus, the included angle between the normal vector for each triangle and the averaged vector can be calculated. The reason for this calculation is to execute a statistics study of the included angles. As mentioned above, for a curled leaf, each normal vector is apt to have a larger included angle with the averaged vector, compared to a more planar leaf. Hence, the curvature of the leaf is perceived and the drought stress is predicted, accordingly. The included angle statistics for some selected planar and curled leaves are shown in Figure 5 and Figure 6, respectively. The horizontal axis represents 


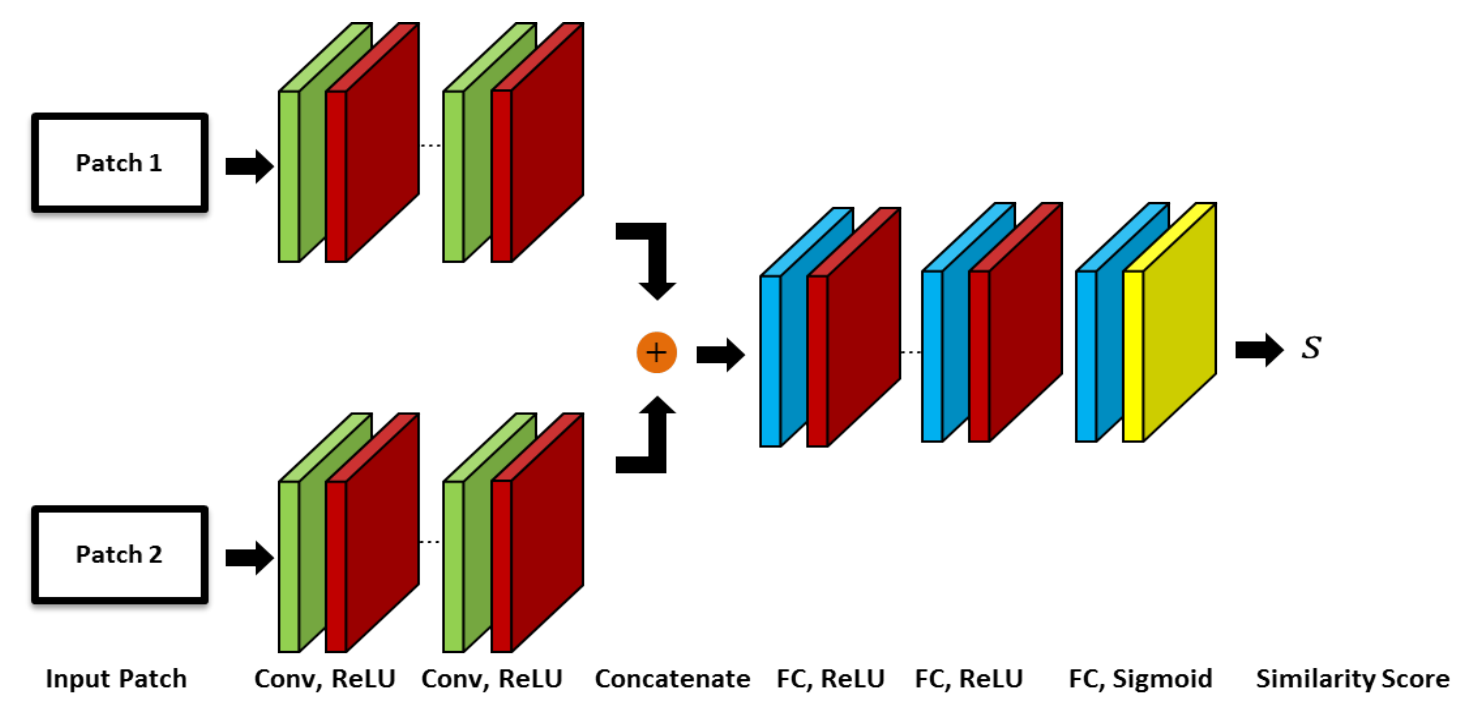

Figure 1. The accurate architecture of MC-CNN (Xia et al., 2019)

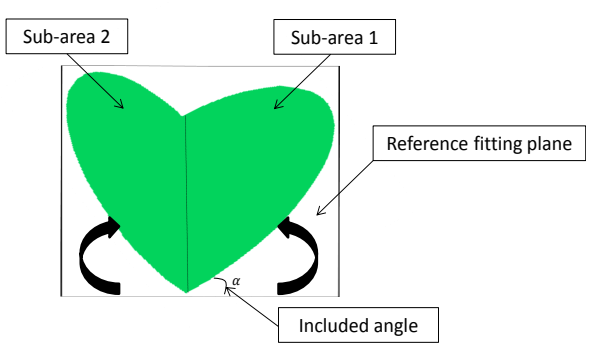

Figure 2. Structure of a curled leaf
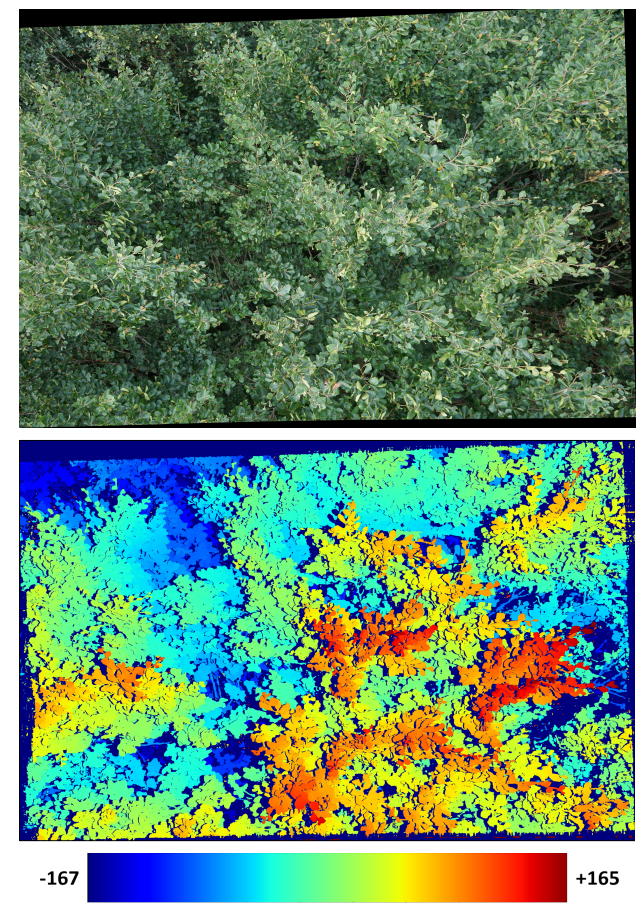

Figure 3. The master epipolar image and the disparity map

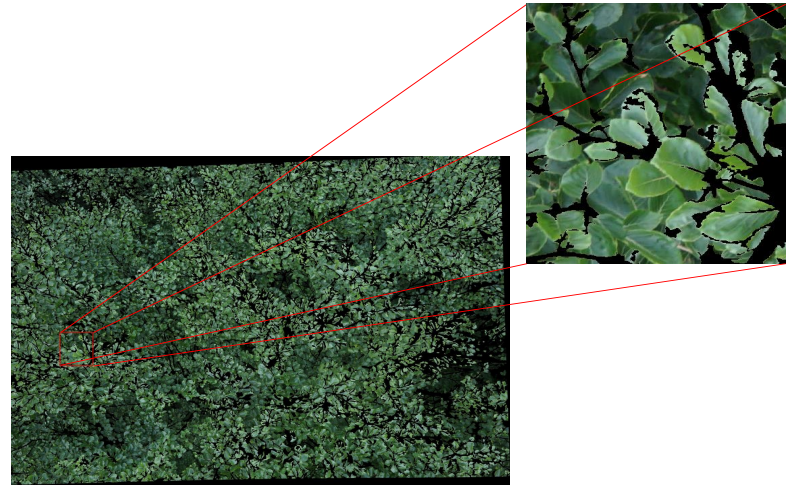

Figure 4. The extracted leaves area with one part highlighted in detail

the range of the included angle, while the vertical axis is the corresponding percentages.

From the figures, it is found that planar leaves have a mean of included angles in the range of 19 - 32 degree, while for curled leaves, the mean is larger, in the range of $37-46$ degree. The difference can clearly provide clues about the curvature of the leaves. In total, 15 planar and 15 curled leaves are selected, as recorded in Table 2, which provides a better distinction between planar and curled leaves. The minimum, median, and maximum of the mean included angles are calculated for the two categories of leaves, respectively.

\begin{tabular}{|c|c|c|}
\hline \multirow{2}{*}{} & \multicolumn{2}{|c|}{ Mean included angle } \\
\cline { 2 - 3 } & Planar & Curled \\
\hline min & $18.15^{\circ}$ & $36.94^{\circ}$ \\
median & $26.30^{\circ}$ & $40.06^{\circ}$ \\
max & $32.72^{\circ}$ & $54.53^{\circ}$ \\
\hline
\end{tabular}

Table 2. Mean included angle statistics for planar and curled leaves

\section{CONCLUSION}

Tree drought stress detection is essential in forest management. $3 \mathrm{D}$ modelling of trees based on dense matching is a proper solution which provides an intuitive representation of the crown's geometric properties. The occlusion caused by 

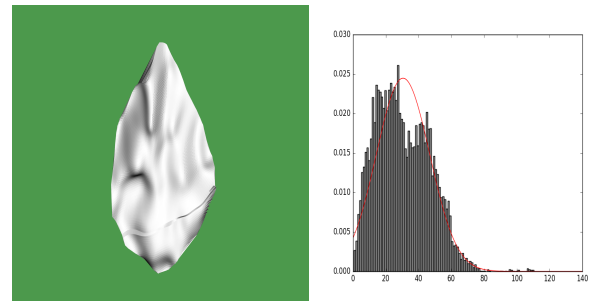

(a) Mean is $30.53^{\circ}$
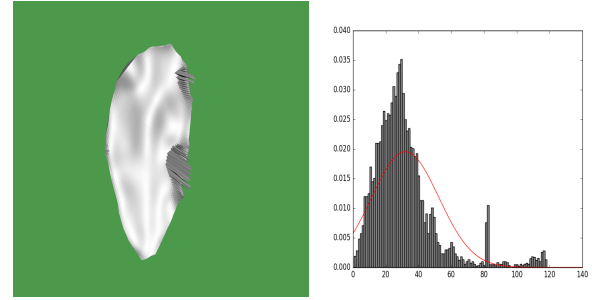

(c) Mean is $31.92^{\circ}$
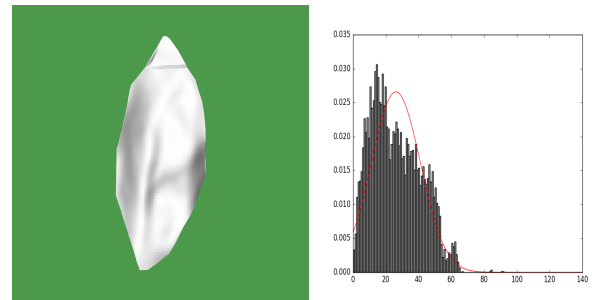

(e) Mean is $26.16^{\circ}$
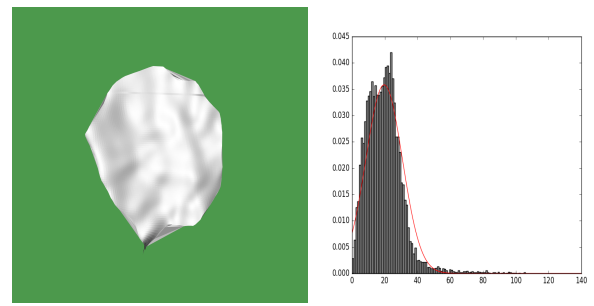

(g) Mean is $19.62^{\circ}$
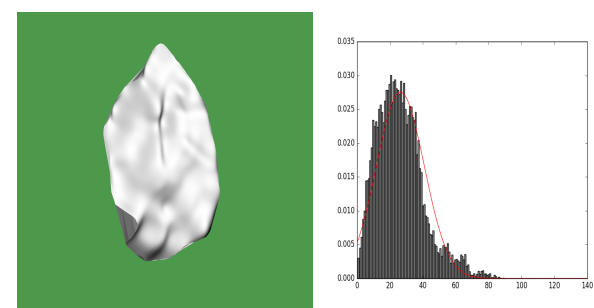

(b) Mean is $26.30^{\circ}$
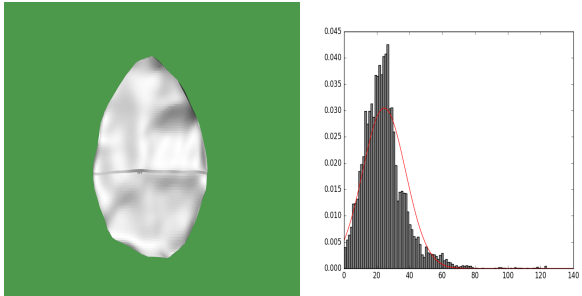

(d) Mean is $24.50^{\circ}$
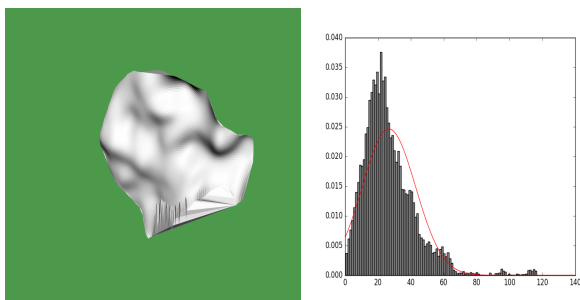

(f) Mean is $26.63^{\circ}$
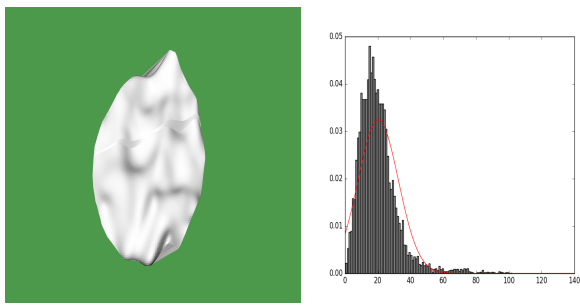

(h) Mean is $20.30^{\circ}$

Figure 5. The included angle statistics of 8 planar leaves

overlapping leaves, nevertheless, seriously influences the matching especially for the trees with dense crown structure.

SGM based on MC-CNN is able to provide high-quality 3D models of leaves. Based on the generated models, the deformation of the leaves can be explored. Thus, the drought status of the tree is better predicted.

In the future, a well-performed segmentation algorithm should be designed to extract leaves and achieve an automatic processing flow. Furthermore, multi-viewed dense matching is promising to achieve denser point clouds for better leaf representation. Multiple images with varying view angles are capable of alleviating the occlusion problem, however, the time for acquiring data should be controlled to limit the environmental effect, such as wind which may swing the branches, causing inconsistency among images.

\section{REFERENCES}

Ahmed, O.S., Franklin, S.E., Wulder, M.A., 2014. Integration of lidar and landsat data to estimate forest canopy cover in coastal British Columbia. Photogrammetric Engineering \& Remote Sensing, 80(10), 953-961.

Bhandare, A., Bhide, M., Gokhale, P., Chandavarkar, R., 2016. Applications of Convolutional Neural Networks. International Journal of Computer Science and Information Technologies (IJCSIT), 7, 2206-2215.

Bromley, J., Bentz, J.W., Bottou, L., Guyon, I., LeCun, Y., Moore, C., Saeckinger, E., Shah, R., 1993. Signature verification using a siamese time delay neural network. International Journal of Pattern Recognition and Artificial Intelligence, 7(4), 669-688.

Calders, K., Origo, N., Burt, A., Disney, M., Nightingale, J., Raumonen, P., Åkerblom, M., Malhi, Y., Lewis, P., 2018. Realistic forest stand reconstruction from terrestrial LiDAR for radiative transfer modelling. Remote Sensing, 10(6), 933.

Delaunay, B., 1934. Sur la sphère vide. A la mémoire de Georges Voronoi. Bulletin de l'Académie des Sciences de l'URSS, Classe des sciences mathématiques et naturelles, 6 , 793-800. 

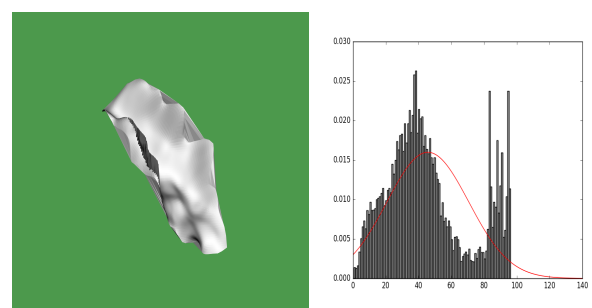

(a) Mean is $45.68^{\circ}$
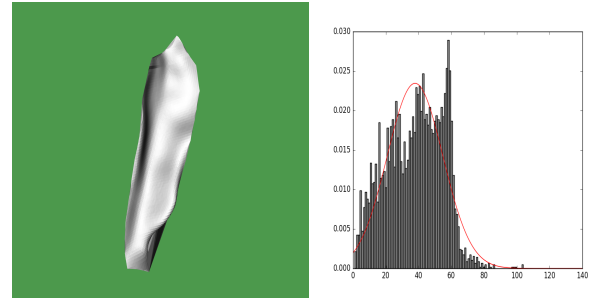

(c) Mean is $37.87^{\circ}$
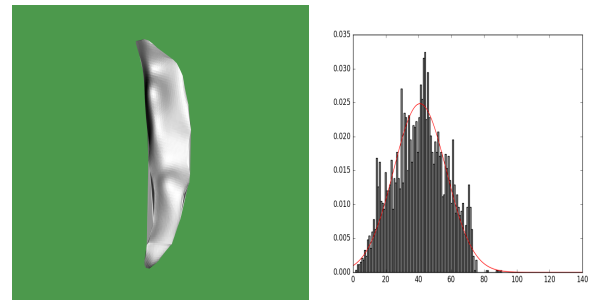

(e) Mean is $40.87^{\circ}$
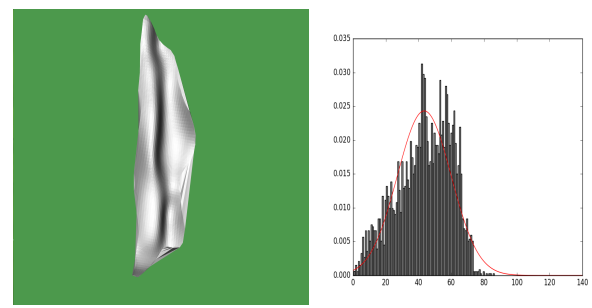

(g) Mean is $43.48^{\circ}$
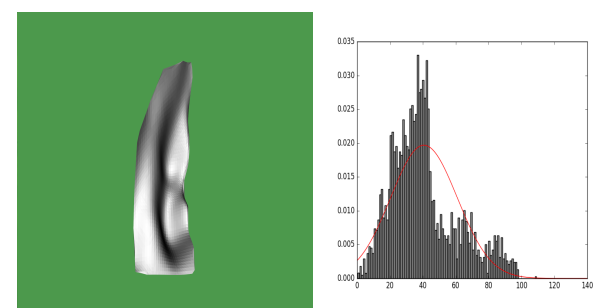

(b) Mean is $40.63^{\circ}$
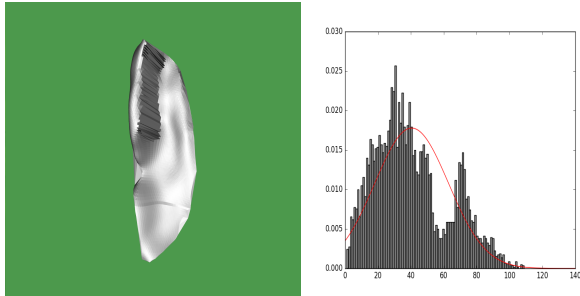

(d) Mean is $40.47^{\circ}$
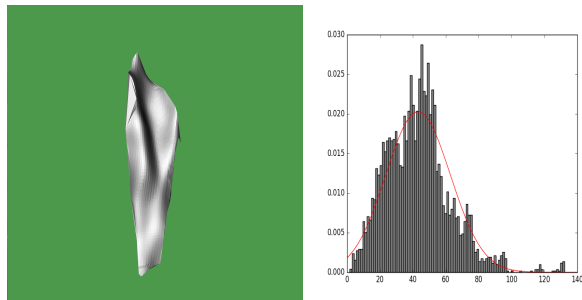

(f) Mean is $43.14^{\circ}$
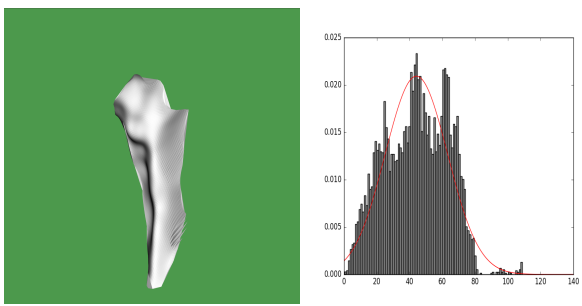

(h) Mean is $44.20^{\circ}$

Figure 6. The included angle statistics of 8 curled leaves

Freeman, M.P., Stow, D.A., Roberts, D.A., 2016. Object-based image mapping of conifer tree mortality in San Diego county based on multitemporal aerial ortho-imagery. Photogrammetric Engineering \& Remote Sensing, 82(7), 571-580.

Gatziolis, D.L., Lienard, J.F., Vogs, A., Strigul, N.S., 2015. $3 \mathrm{D}$ tree dimensionality assessment using photogrammetry and small unmanned aerial vehicles. PLoS ONE, 10(9), e0137765.

Geiger, A., Lenz, P., Stiller, C., Urtasun, R., 2013. Vision meets robotics: the KITTI dataset. International Journal of Robotics Research, 32(11), 1231-1237.

Gitelson, Anatoly A, Kaufman, Yoram J, Stark, Robert, Rundquist, Don, 2002. Novel algorithms for remote estimation of vegetation fraction. Remote sensing of Environment, 80, $76-87$.

Hang, R., Liu, Q, Hong, D., Ghamisi, P., 2019. Cascaded Recurrent Neural Networks for Hyperspectral Image Classification. arXiv preprint arXiv:1902.10858.

Hirschmueller, H., 2008. Stereo processing by semiglobal matching and mutual information. IEEE Transactions on Pattern Analysis and Machine Intelligence, 30(2), 328-341.
Hirschmueller, H., Scharstein, D., 2009. Evaluation of stereo matching costs on images with radiometric differences. IEEE Transactions on Pattern Analysis and Machine Intelligence, 31(9), 1582-1599.

Hong, D., Liu, W., Su, J., Pan, Z., Wang, G., 2015. A novel hierarchical approach for multispectral palmprint recognition. Neurocomputing, 151, 511-521.

Hong, D., Yokoya, N., Chanussot, J., Zhu, X.X., 2018. An augmented linear mixing model to address spectral variability for hyperspectral unmixing. IEEE Transactions on Image Processing, 28, 1923-1938.

Kaufman, YORAM J, 1989. The atmospheric effect on remote sensing and its correction. Theory and application of optical remote sensing, 336-428.

Krizhevsky, A., Sutskever, I., Hinton, G.E., 2012. Imagenet classification with deep convolutional neural networks. Advances in Neural Information Processing Systems, 1106-1114.

Lau Sarmiento, A.I., Bartholomeus, H., Herold, M., Martius, C., Malhi, Y., Bentley, L.P., Raumonen, P., 2015. Application of 
terrestrial LiDAR and modelling of tree branching structure for plant-scaling models in tropical forest trees. In Proceedings of SilviLaser 2015, The 14th Conference on Lidar Applications for Assessing and Managing Forest Ecosystems, La Grande Motte, France.

LeCun, Y., Bottou, L., Bengio, Y., Haffner, P., 1998. Gradient-based learning applied to document recognition. Proceedings of the IEEE, 86(11), 2278-2324.

Levin, S.A., 1999. Fragile Dominion: Complexity and the Commons. Perseus Books Group, Massachusetts, USA.

Menze, M., Geiger, A., 2015. Object scene flow for autonomous vehicles. IEEE Conference on Computer Vision and Pattern Recognition, Boston, Massachusetts, USA, 3061-3070.

Metz, J., Seidel, D., Schall, P., Scheffer, D., Schulze, E.D., Ammer, C., 2013. Crown modeling by terrestrial laser scanning as an approach to assess the effect of aboveground intra-and interspecific competition on tree growth. Forest Ecology and Management, 310, 275-288.

Rosu, A.M., Pierrot-Deseilligny, M., Delorme, A., Binet, R., Klinger, Y., 2015. Measurement of ground displacement from optical satellite image correlation using the free open-source software MicMac. ISPRS Journal of Photogrammetry and Remote Sensing, 100, 48-59.

Scharstein, D., Hirschmueller, H., Kitajima, Y., Krathwohl, G., Nesic, N., Wang, X., Westling, P., 2014. High-resolution stereo datasets with subpixel-accurate ground truth. German Conference on Pattern Recognition, Muenster, Germany.

Scharstein, D., Pal, C., 2007. Learning conditional random fields for stereo. IEEE Computer Society Conference on Computer Vision and Pattern Recognition, Minneapolis, Minnesota, USA.

Scharstein, D., Szeliski, R., 2002. A taxonomy and evaluation of dense two-frame stereo correspondence algorithms. International Journal of Computer Vision, 47(1/2/3), 7-42.

Scharstein, D., Szeliski, R., 2003. High-accuracy stereo depth maps using structured light. IEEE Computer Society Conference on Computer Vision and Pattern Recognition, Madison, Wisconsin, USA, 1, 195-202.

Strigul, N., 2012. Individual-based models and scaling methods for ecological forestry: implications of tree phenotypic plasticity. Sustainable Forest Management (eds J Garcia, J Casero), Rijeka, Croatia, InTech, 359-384.

Tian, J., Schneider, T., Straub, C., Kugler, F., Reinartz, P., 2017. Exploring digital surface models from nine different sensors for forest monitoring and change detection. Remote Sensing, 9(3), 287.

Wu, X., Hong, D., Ghamisi, P., Li, W., Tao, R., 2018. Msri-ccf: Multi-scale and rotation-insensitive convolutional channel features for geospatial object detection. Remote Sensing, 10, 1990.

Wu, Z., Dye, D., Vogel, J., Middleton, B., 2016. Estimating forest and woodland aboveground biomass using active and passive remote sensing. Photogrammetric Engineering \& Remote Sensing, 82(4), 271-281.
Xia, Y.X., d'Angelo, P., Tian, J., Fraundorfer, F., Reinartz, P., 2019. Self-supervised convolutional neural networks for plant reconstruction using stereo imagery. Photogrammetric Engineering \& Remote Sensing.

Xu, P., Zhou, T., Yi, C., Luo, H., Zhao, X., Fang, W., Gao, S., Liu, X., 2018. Impacts of water stress on forest recovery and its interaction with canopy height. International Journal of Environmental Research and Public Health, 15(6), 1257.

Zbontar, J., LeCun, Y., 2016. Stereo matching by training a convolutional neural network to compare image patches. Journal of Machine Learning Research, 17, 1-32. 